\title{
Carbon Fluoride, CFx: Structural Diversity as Predicted by First Principles
}

Cecilia Goyenola, Sven Stafström, Susann Schmidt, Lars Hultman and Gueorgui Kostov

Gueorguiev

\section{Linköping University Post Print}

\section{Tweet}

N.B.: When citing this work, cite the original article.

Original Publication:

Cecilia Goyenola, Sven Stafström, Susann Schmidt, Lars Hultman and Gueorgui Kostov Gueorguiev, Carbon Fluoride, CFx: Structural Diversity as Predicted by First Principles, 2014, The Journal of Physical Chemistry C, (118), 12, 6514-6521.

http://dx.doi.org/10.1021/jp500653c

Copyright: American Chemical Society http://pubs.acs.org/

Postprint available at: Linköping University Electronic Press http://urn.kb.se/resolve?urn=urn:nbn:se:liu:diva-106285 


\title{
Carbon Fluoride, $\mathrm{CF}_{x}$ : Structural Diversity as Predicted by First Principles
}

\author{
C. Goyenola, S. Stafström, S. Schmidt, L. Hultman, and G. K. Gueorguiev* \\ Department of Physics, Chemistry and Biology (IFM), Linköping University, SE-58183 \\ Linköping, Sweden \\ E-mail: gekos@ifm.liu.se
}

Phone: (+46)13282491. Fax: (+46)13137568

KEYWORDS: Density funtional theory, fluorine, nanostructure, thin films, graphene

${ }^{*}$ To whom correspondence should be addressed 


\begin{abstract}
Fluorinated carbon-based thin films offer a wide range of properties for many technological applications that depend on the microstructure of the films. To gain a better understanding on the role of fluorine in the structural formation of these films, $\mathrm{CF}_{x}$ systems based on graphenelike fragments were studied by first principles calculations. Generally, the F concentration determines the type of film that can be obtained. For low F concentrations (up to $\sim 5$ at.\%), films with fullerene-like as well as graphite-like features are expected. Larger F concentrations ( $\geq 10$ at.\%) give rise to increasingly amorphous carbon films. Further increasing the F concentration in the films leads to formation of a polymer-like microstructure. In order to aid characterization of $\mathrm{CF}_{x}$ systems generated by computational methods, a statistical approach is developed.
\end{abstract}

\title{
Introduction
}

Carbon-based thin solid films constitute a family of materials offering many technologically important properties, such as high hardness, high elastic modulus, low friction coefficient, and chemical inertness. They can form predominantly amorphous films, such as diamond-like carbon (DLC) films,${ }^{1-3}$ tetrahedral amorphous carbon (ta-C) films, ${ }^{2}$ and also as inherently nanostructured films as in the case of fullerene-like (FL) carbon films. ${ }^{4}$

Unlike graphite and diamond, where carbon atoms exist in only one hybridization state $\left(\mathrm{sp}^{2}\right.$ and $\mathrm{sp}^{3}$, respectively), in carbon-based thin films carbon atoms with different hybridization coexist. The relative content of $\mathrm{sp}^{3}$ to $\mathrm{sp}^{2}$ hybridized carbon atoms gives rise to a wide diversity of films microstructure, and consequently, different combinations of properties. While DLC and ta-C films exhibit a high ratio of $\mathrm{sp}^{3}$ to $\mathrm{sp}^{2}$ bonded carbon, ${ }^{2,3,5,6}$ the nanostructured FL films represent the opposite case with predominantly $\mathrm{sp}^{2}$ hybridized carbon atoms. ${ }^{4,7}$ As a consequence, DLC and ta-C films show higher to comparable hardness than FL films, but lower elasticity.

In order to tailor the properties of nanostructured carbon-based thin films for desired technological applications, other elements such as nitrogen, ${ }^{4,8-10}$ phosphorus, ${ }^{11-13}$ sulfur ${ }^{8,14-16}$ and 
fluorine ${ }^{17-25}$ are often incorporated in the carbon matrix at concentrations between 0-30 at.\%. Considering the list of possible heteroatom incorporation, fluorine attracts special attention due to its substantial chemical differences compared to the other already worked out elements (N, P, S). Fluorine possesses more valence electrons and can only form a single bond with carbon. It also exhibits the highest electronegativity and one of the highest reactivities among all the elements in the Periodic Table. Hence, compared to carbon and to the previously mentioned elements, fluorine introduces quite distinct chemical and bonding features to the films.

Fluorinated carbon-based $\left(\mathrm{CF}_{x}\right)$ thin films have been synthesized by different variants of chemical vapor deposition ${ }^{17-21}$ as well as by physical vapor deposition. ${ }^{22-27} \mathrm{CF}_{x}$ films display a low dielectric constant and a low refractive index,${ }^{17,19,20}$ moderate hardness ${ }^{21,23,25}$ as well as low friction coefficients, high wear resistance, ${ }^{23}$ and biocompatibility. ${ }^{21}$ These properties make them suitable for electrical, tribological, and biomedical applications. Even though the specific characteristics and properties of $\mathrm{CF}_{x}$ films are dependent on the deposition process, there is a general agreement that the F concentration determines to a significant extent the nature of the microstructure and thus the properties, i.e., polymer-like ${ }^{1}$, diamond-like, and fullerene-like in order of decreasing F content. ${ }^{25,27}$ Nevertheless, a complete atomistic understanding of the role and impact of fluorine in the microstructure of as-deposited $\mathrm{CF}_{x}$ films is still lacking and it is indispensable for tailoring their structure and properties by guiding the deposition process.

In this context, computational methods appear as a very valuable tool. In order to study thin films formed by vapor phase deposition techniques, we have developed the synthetic growth concept (SGC) based on the density functional theory (DFT). ${ }^{12,28,29}$ The SGC is an extensive approach that describes the properties of graphene-like pieces and nanotemplates for nanostructured materials together with precursors and their interaction for the film formation.

In our previous work carried out by $\mathrm{SGC},{ }^{25,30}$ we studied the substitution of a $\mathrm{C}$ atom by a $\mathrm{F}$ atom in graphene-like model systems. This study resulted in a classification of structural

\footnotetext{
${ }^{1}$ Polymer-like refers to chain structures that form in the film during vapor phase deposition. These chains resemble short polymeric chains, but due to the stochastic nature of the deposition process, they do not consist in rigorously repetitive $\mathrm{C}_{m} \mathrm{~F}_{n}$ units.
} 
patterns in F-doped graphene-like networks (0-7 at.\% of F): (i) large $\mathrm{N}$ members rings $(\mathrm{N}=8-12)$, (ii) branching of graphene sheets, and (iii) $\mathrm{C}-\mathrm{F}$ bond rotation at the edge of a model system. Based on the structural effects caused by the substitution of one $\mathrm{F}$ atom, it was predicted that FL-CF may be synthesized for F contents lower than 10 at.\%. Higher F concentrations were expected to lead to higher concentration of defects tending to amorphization and to polymerization. ${ }^{25,30}$

In pursuit of further understanding the structure of fluorinated carbon-based thin films and the mechanism of their formation, in this work we explore the structural and energetic implications of different concentrations of fluorine in graphene-like sheets. While in our previous work we studied the effects of a substitutional fluorine atom in pure carbon model systems, now we approach the structural evolution of essentially binary systems $\mathrm{CF}_{x}$ with fluorine contents ranging between 0 at.\% and 30 at.\%. This new approach to the $\mathrm{CF}_{x}$ structural problem demanded the adoption of a significant number (125) of newly assembled model systems with different fluorine concentrations. Such diversity permitted us to carry out a systematic statistical analysis for $\mathrm{CF}_{x}$ structural classification.

\section{Computational Details}

A systematic study of the structural implications of substitutional $\mathrm{F}$ atoms in graphene-like networks was carried out by performing geometry optimizations followed by assessment of the energy cost for F substitution. The study was performed using graphene-like model systems, in which $\mathrm{C}$ atoms were sequentially substituted by F atoms. Substitutions were implemented in two ways: (i) $\mathrm{C}$ atoms were substituted by $\mathrm{F}$ atoms one at a time in a previously relaxed structure, thus increasing the $\mathrm{F}$ content of the system in a step-wise manner; and (ii) a number of $\mathrm{C}$ atoms were substituted by $\mathrm{F}$ atoms instantly in a relaxed pure carbon model system, i.e., increasing its $\mathrm{F}$ content in one step only.

The size of the model systems adopted for the present studies is carefully chosen: (i) to be large enough to be able to incorporate different types of defects at different sites, including with 
respect to the model system's edge; and (ii) to be sized moderately enough to permit a statistical and systematic analysis of a quite wide range of $\mathrm{CF}_{x}$ systems at a sophisticated ab-initio level of theory with demanding yet acceptable computational cost.

The cohesive energy per atom $\left(E_{c o h / a t}\right)$ was calculated for all model systems. $E_{c o h / a t}$ is defined as the energy required to break the system into isolated atoms normalized by the total number of $\mathrm{C}$ and $\mathrm{F}$ atoms:

$$
E_{c o h / a t}=\left|\frac{E_{\text {system }}-n_{H} \times E_{H}-n_{C} \times E_{C}-n_{F} \times E_{F}}{n_{C}+n_{F}}\right|,
$$

where $E_{\text {system }}$ is the total energy of the model system, $n_{H}, n_{C}$, and $n_{F}$ are the number of $\mathrm{H}, \mathrm{C}$, and $\mathrm{F}$ atoms, and $E_{H}, E_{C}$, and $E_{F}$ are the energies of the corresponding free atoms in ground state, respectively.

The framework adopted for the present simulations is DFT within the generalized gradient approximation (GGA) as implemented in the Gaussian 09 code. ${ }^{31}$ The results reported herein were obtained using the PW91 ${ }^{32}$ exchange and correlation functionals with the $6-31 \mathrm{G}^{* *}$ basis set augmented with polarization functions. For comparative purposes, some of the simulations were repeated using the $\mathrm{B} 3 \mathrm{LYP}^{33}$ hybrid functional. Both functionals have been proved to give an appropriate description, structurally and electronically, of FL thin films ${ }^{12,28}$ and similar covalent systems. ${ }^{34-37}$ In order to assess the effect of dispersion interaction, Grimme's functional ${ }^{38}$ was also used for test optimizations of some of the largest systems resulting from multistep structural evolution. The influence of the basis set was evaluated by also using the $6-31++\mathrm{G}^{* *}$ basis set which includes diffuse functions. The results obtained with B3LYP, Grimme's functional, and 6$31++\mathrm{G}^{* *}$ do not differ significantly from those obtained at the PW91/6-31G** level of theory and are not reported here. 


\section{Results and Discussion}

\section{Incorporation of $\mathbf{F}$ atoms in graphene-like networks}

The graphene-like fragments presented in Fig. 1 were adopted as initial model systems to study the structural and energetic implications of substitutional $\mathrm{F}$ in graphene-like networks and include a pure hexagonal graphene flake (Fig. 1 (a)), a graphene-like system containing a single pentagon defect (Fig. 1 (b)), and another one containing a Stone-Wales defect (Fig. 1 (c)). The initial stoichiometry for all three of these systems was $\mathrm{C}_{42} \mathrm{H}_{16}$. The study was carried out substituting $\mathrm{C}$ atoms by $\mathrm{F}$ atoms, thus increasing their $\mathrm{F}$ content from 0 at.\% to 30 at.\% (as explained in section "Computational Details"). For each model system, the sequence of substitution of $\mathrm{C}$ atoms by $\mathrm{F}$ atoms was determined randomly and alternative substitutional patterns were considered. This procedure resulted in 125 different $\mathrm{CF}_{x}$ network structures divided in 14 groups according to their F concentration.
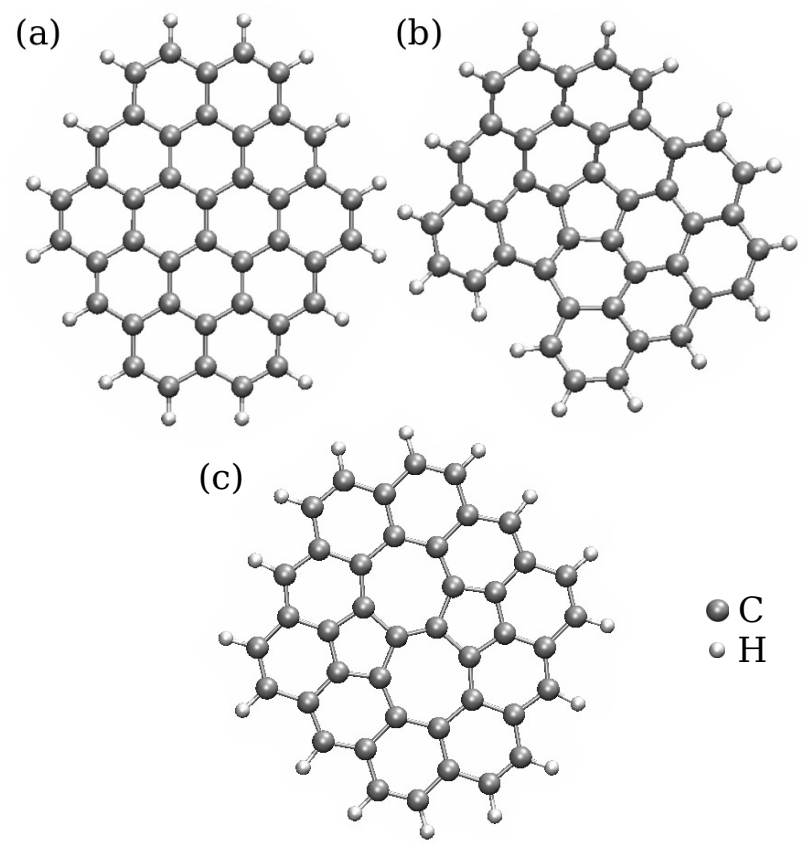

Figure 1: Model systems adopted for the study of the effect of substitutional fluorine in a graphenelike network: (a) a pure hexagonal network; (b) model system containing a single pentagon defect; and (c) a model system containing a Stone-Wales defect (combination of a pentagon and a heptagon). 
Fig. 2 (a, b, c, d) display $\mathrm{CF}_{x}$ structures with typical morphologies at different $\mathrm{F}$ concentrations resulting from the substitution of $\mathrm{C}$ by $\mathrm{F}$ atoms in the model systems shown in Fig. 1. It was found that relatively low F concentrations (of about 5 at.\%) already lead to a rapid disintegration of the structural characteristics of the $\mathrm{sp}^{2} \mathrm{C}$ network. As the $\mathrm{F}$ content is further increased, the $\mathrm{CF}_{x}$ networks become progressively more disrupted and usually, only a small fraction of them retain regularly looking defects, such as large rings and branching. ${ }^{30}$ Features like interlocking and amorphousness strongly prevail.

(a)

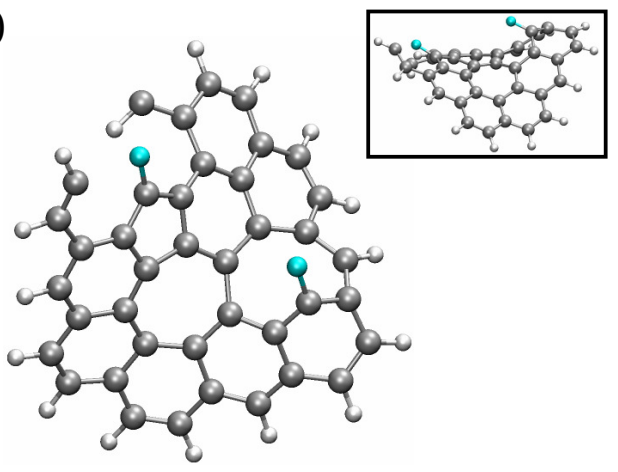

5.0 at. $\%$

(c)

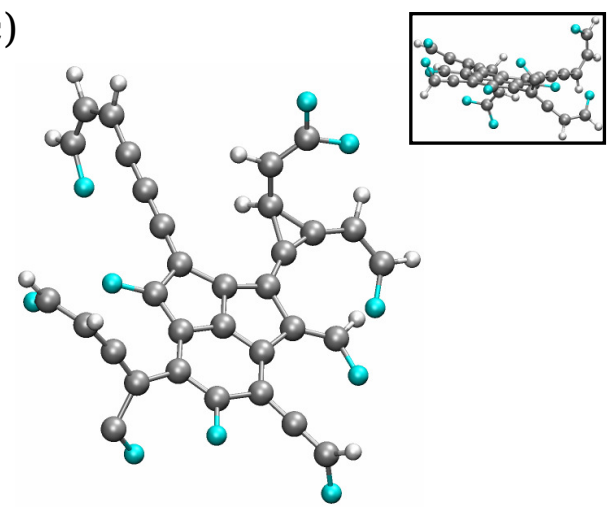

24.0 at. $\%$ (b)

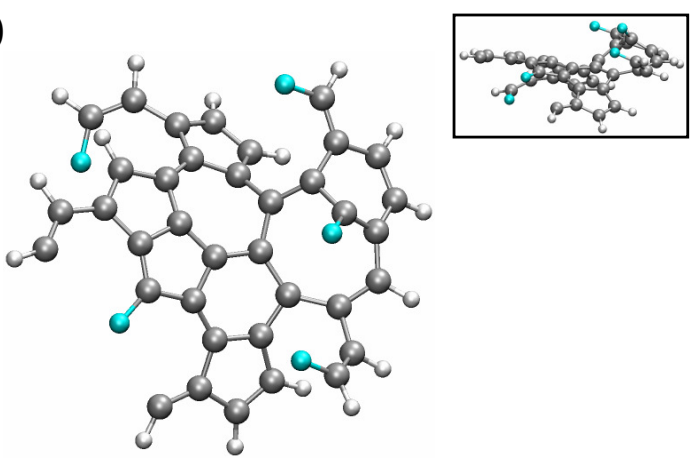

12.0 at. $\%$

○ C

- $\mathrm{F}$

- $\mathrm{H}$

Figure 2: Typical $\mathrm{CF}_{x}$ structures resulting from the substitution of $\mathrm{C}$ atoms by $\mathrm{F}$ atoms in the model systems shown in Fig. 1. The insets correspond to different views of each structure. The corresponding F content is indicated at the bottom of each panel.

These observations are explained considering the fact that $\mathrm{F}$ is chemically very different from $\mathrm{C}$, and thus, the changing impact of its incorporation in a graphene-like network is substantial. F atoms can only accept one electron, hence, F cannot be integrated into the two-dimensional net- 
work as it is the cases of substitutional $\mathrm{N},{ }^{28} \mathrm{P}^{12,39}$ or $\mathrm{S},{ }^{16,40}$ where the heteroatom takes the place of a $\mathrm{C}$ atom preserving, to a significant extent, the integrity of the network. Instead, incorporated F tends to stick out of the network and to provoke a local disruption, leading to characteristic patterns such as large rings and branching. ${ }^{30}$ As the amount of F incorporated in the network increases, these disruption patterns densify and start to interact with each other. Some of them prevail (i.e., preserve their initial structural identity), while others recombine to form structural features at a larger size scale.

As a consequence of the fact that $\mathrm{F}$ atoms have the ability to disrupt the carbon network when incorporated at a $\mathrm{C}$ site, the substitution of several $\mathrm{C}$ atoms by $\mathrm{F}$ atoms at neighboring sites leads to opening holes in the network and, ultimately, may cause breaking the network into fragments. Since the mechanisms of network fragmentation are not in the focus of this work, the only restriction imposed to the sequential substitution process was that the evolved model systems remain linked networks.

Relevant structural information can be obtained by studying how the total number of middle size rings (MSR - rings with 5, 6, and 7 atoms), the number of $\mathrm{C}$ atoms with 3 neighbors (\#C3n), and the cohesive energy per atom $\left(E_{c o h / a t}\right)$ vary with the $\mathrm{F}$ content in the $\mathrm{CF}_{x}$ systems. These three quantitative characteristics were determined for each of the aforementioned relaxed systems, and then plotted as a function of the systems F content. The plots in Fig. 3 (a, b, c) show these dependencies, respectively.

The variation in the total number of MSR describes how the graphene-likeliness of the networks vary with $\mathrm{F}$ content. The reason to consider rings with 5, 6 and 7 atoms is obvious: graphene networks are inherently hexagonal networks, but they may also include structural defects such as pentagons and heptagons while still preserving their graphene-like character. In addition, the \#C3n relates qualitatively to $\mathrm{sp}^{2}$ hybridization, and can also be directly related to the graphene-likeliness of the networks. The plot in Fig. 3 shows how the total number of MSR (Fig. 3 (a)) and \#C3n (Fig. 3 (b)) decrease with the increasing F content. This means that the graphene-like character of the networks studied is progressively lost with increasing F content, to the point that above 10 at. $\%$ 

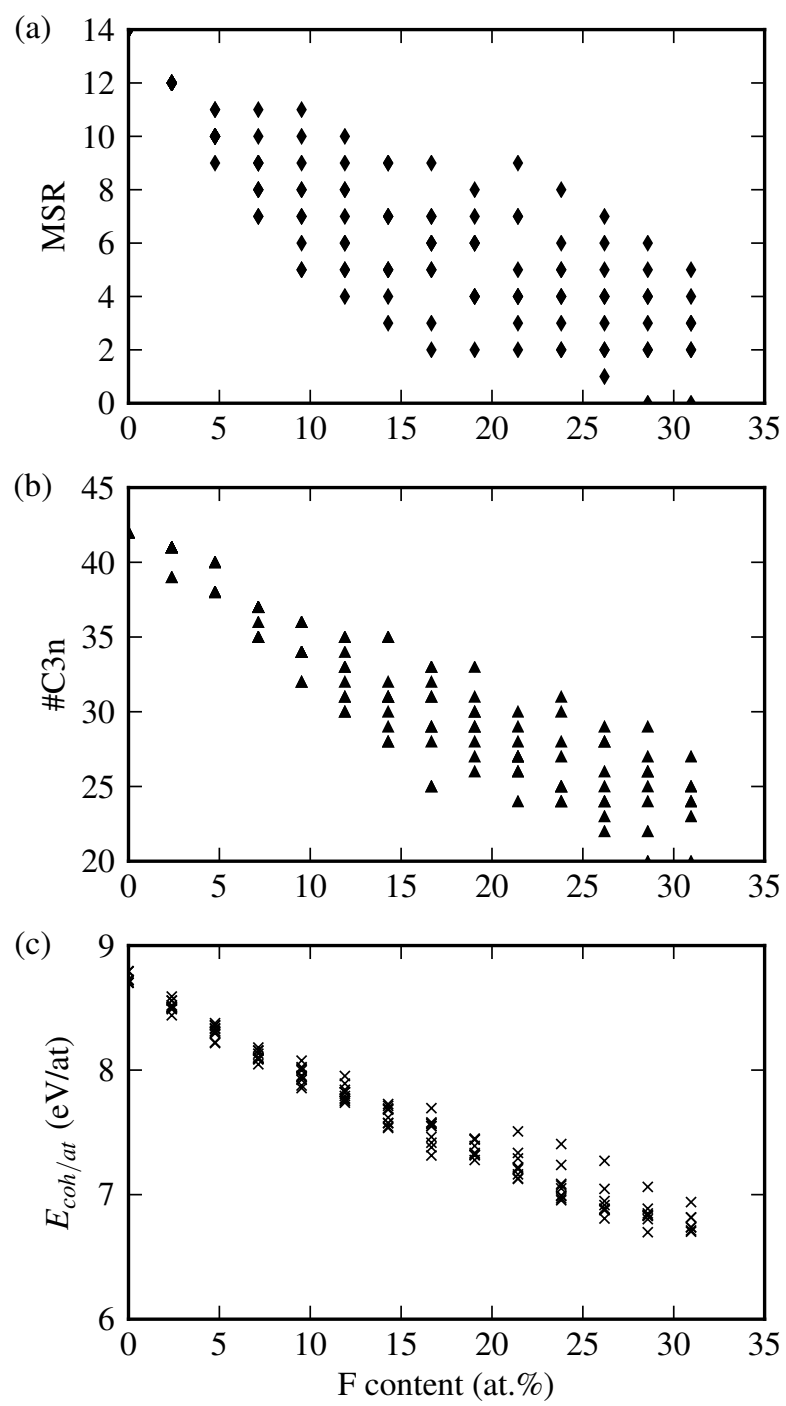

Figure 3: (a) Number of middle size rings (MSR), (b) number of $\mathrm{C}$ atoms with three neighbors (\#C3n), (c) cohesive energy per atom $\left(E_{c o h / a t}\right)$ as a function of the $\mathrm{F}$ content for the whole ensemble of $125 \mathrm{CF}_{x}$ systems studied. 
of $\mathrm{F}$, the $\mathrm{CF}_{x}$ model systems have no resemblance to the original graphene-like templates, (c.f., Fig. 1, and Fig. 2).

Fig. 2 also suggests that, besides the total $\mathrm{F}$ content, the distribution of $\mathrm{F}$ atoms within the $\mathrm{C}$ based network has a decisive impact on the resulting conformations. Depending on the distribution of $\mathrm{F}$ atoms, the following structural trends may be favored: (i) chain prevalence (e.g., Fig. 2 (c)); (ii) rings arranged in a strongly interlocked pattern (e.g., Fig. 2 (d)); (iii) any combination of chains and interlocked rings. These results, together with the fact that $\mathrm{F}$ atoms support a single bond indicate that $\mathrm{F}$ segregation is not an issue for $\mathrm{CF}_{x}$ compounds. Instead, as it is also the case of $\mathrm{CN}_{x}$ thin solid films, ${ }^{41}$ regions with considerable variation of the local concentration of the heteroatom (in this case: F) can occur, mostly depending on the types of precursors available during deposition. ${ }^{25-27}$

These structural features are correlated with the dependence of cohesive energy per atom on the F concentration (Fig. 3 (c)). The incorporation of F atoms in a graphene-like network reduces the $E_{c o h / a t}$, which translates into a relative decrease in overall structural stability. This can be explained by the fact that every $\mathrm{F}$ atom incorporated in the network leads to disruptions of the graphene-network and, in many cases, causes dangling bonds on adjacent carbon atoms.

The cohesive energy trend (Fig. 3 (c)) also suggests that as the F content increases, the dispersion in the $E_{c o h / a t}$ values for the different F concentrations widens. To illustrate this tendency, the cohesive energy differences $\left(\Delta E_{c o h / a t}\right)$ between the most stable $\mathrm{CF}_{x}$ conformation (i.e., the one exhibiting minimum $\left.E_{c o h / a t}\right)$ and the remaining $\mathrm{CF}_{x}$ conformations, i.e., the dispersion of $E_{c o h / a t}$, were calculated for each F content considered. This cohesive energy dispersion is plotted in Fig. 4. The trend in $E_{c o h / a t}$ dispersion shows a relatively slow, but steady increase with the F content up to 15 at.\% $\left(0 \leq \Delta E_{c o h / a t} \leq 0.25 \mathrm{eV}\right)$. Beyond 15 at.\% of $\mathrm{F}$, the range of $E_{c o h / a t}$ values considerably increases, after which it remains approximately constant $\left(0 \leq \Delta E_{c o h / a t} \leq 0.50 \mathrm{eV}\right)$.

This energetic trend mirrors the tendency found for the structural changes of the systems with increasing F content, shown in Fig. 2. Low F concentrations $(\sim 5$ at. \%) are associated with a small amount of disruptions in the graphene-like systems, including mild bending of the atomic sheets. 


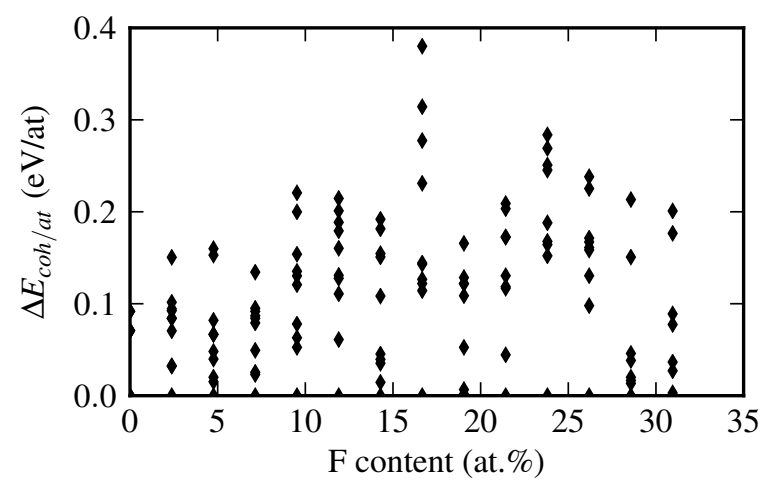

Figure 4: Dispersion in cohesive energy per atom $\left(\Delta E_{c o h / a t}\right)$ for the $\mathrm{CF}_{x}$ model systems.

Structural features at intermediate F concentrations (between $\sim 5$ at. $\%$ and $\sim 15$ at. $\%$ ) are characterized by a partially preserved graphene-like structure containing larger rings and/or short chains, increasing the variety of conformations and, consequently, the dispersion in $E_{c o h / a t}$. Higher F concentrations $(>15$ at.\%) result in networks formed by rings of different sizes tending to interlocked configurations, as well as networks with chains ramifications. In other words, $\mathrm{CF}_{x}$ films with $\mathrm{F}$ contents higher than 15 at.\% would show a variety of structures from DLC (interlocked configurations) to polymer-like (chain ramifications). Such a large variety of conformations correlates well with an even higher dispersion in the cohesive energies. These results for the $\mathrm{CF}_{x}$ structural dependence on the $\mathrm{F}$ content are in good agreement with experimental observations, the characterization of $\mathrm{CF}_{x}$ thin films deposited by high-power impulse magnetron sputtering showed a tendency from graphite-like to polymer-like with increasing F content. ${ }^{25-27}$

\section{Statistical analysis of $\mathbf{C F}_{x}$ systems}

Essential mechanical properties of C-based thin films, such as hardness and wear resistance, strongly depend on the short range order of the atoms in the compound and the bonding environment surrounding each $\mathrm{C}$ atom. Hence, it is important to describe and quantify these short range structural features.

With this aim, the type of bonds in which the $\mathrm{C}$ atoms participate were studied. The number of $\mathrm{C}$ atoms bonded to two (\#C2n), three (\#C3n), and four (\#C4n) atoms was determined for all 
relaxed $\mathrm{CF}_{x}$ model systems (introduced in the previous subsection) as an estimation of the content of $\mathrm{sp}^{3}, \mathrm{sp}^{2}$, and sp hybridized $\mathrm{C}$ atoms, respectively. Fig. 5 (a, b, c) systematizes these statistics for the different $\mathrm{F}$ concentrations, showing the number of $\mathrm{C}$ atoms bonded to $n$ atoms $(n=2-4)$ as a percentage of the total number of $\mathrm{C}$ atoms in the corresponding $\mathrm{CF}_{x}$ model system. The case of $\mathrm{C}$ atoms with only one neighbor is excluded from the discussion since they are not present in any of the $\mathrm{CF}_{x}$ model systems considered.
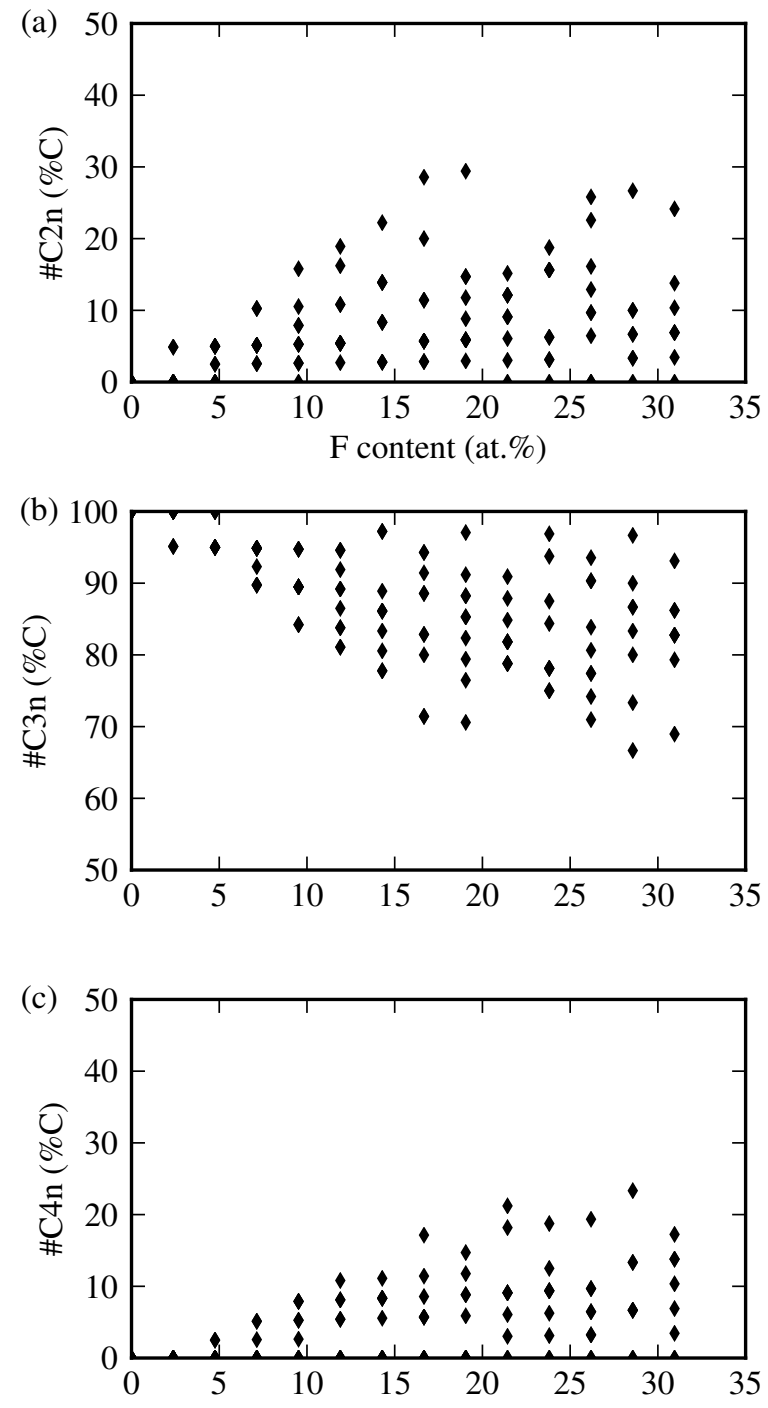

Figure 5: Evolution of the total number of $\mathrm{C}$ atoms with (a) 2 (\#C2n), (b) 3 (\#C3n), and (c) 4 (\#C4n) neighbors expressed as a percentage of the total number of $\mathrm{C}$ atoms for all $\mathrm{CF}_{x}$ model systems considered.

As seen from Fig. 5 (b), the number of $\mathrm{C}$ atoms with three neighbors (\#C3n) decreases when 
the $\mathrm{F}$ content increases. For $\mathrm{F}$ contents up to $\sim 15$ at. $\%$, this value decreases rapidly, and is accompanied by an increase in the number of $\mathrm{C}$ atoms with two and four neighbors (Fig. 5 (a) \#C2n, and Fig. 5 (c) - \#C4n, respectively). These results indicate how the incorporation of $\mathrm{F}$ atoms into the graphene network promotes a change in the hybridization of the $\mathrm{C}$ atoms. These results also agree very well with the observation that the incorporation of $\mathrm{F}$ in a graphene network gradually degrades its three-coordinated $\mathrm{sp}^{2}$-hybridized planar structure, by promoting interlocking and amorphousness (previous subsection, and Ref. ${ }^{30}$ ).

At higher F contents ( $>15$ at.\%), a noticeable dispersion in the number of $\mathrm{sp}^{2}$-hybridized C atoms is observed, indicating that a large variety of structures may be found for $\mathrm{CF}_{x}$ systems with F contents over 15 at.\%, and corroborating the previous discussion in "Incorporation of F atoms in graphene-like networks" subsection.

It is noteworthy that for many of the systems with high $\mathrm{F}$ content ( $>15$ at.\%), a large portion of the $\mathrm{sp}^{2}$-hybridized $\mathrm{C}$ atoms are bonded to one or even two $\mathrm{F}$ atoms. The second case is the simplest to understand since a $\mathrm{CF}_{2}$ group with an $\mathrm{sp}^{2}$-hybridized $\mathrm{C}$ atom can bond with only one $\mathrm{C}$ atom of the network, thus acting as a termination group for a chain or as a substituent of a ring (see Fig. 6). The first case (i.e., a $\mathrm{C}$ atom bonded to one $\mathrm{F}$ atom), on the other hand, has more bonding options and gives rise to a variety of structures. The $\mathrm{C}$ and $\mathrm{F}$ atoms form a $\mathrm{CF}$ group that can be bonded to two $\mathrm{C}$ atoms belonging to the network (see Fig. 6). In particular, if several $\mathrm{CF}$ groups bond together, they would originate the formation of chains or form rings at the edge of the network. The promotion of chains leads to polymer-like structures while the formation of rings at the network's edge may lead to interlocked conformations or creation of grain boundaries.

To analyze further the structure of the optimized model systems, the distribution of $\mathrm{C}-\mathrm{C}$ distances $^{2}\left(d_{C-C}\right)$ was calculated for all relaxed systems. In Fig. 7 (a, b, c, d) are shown the histograms representing the distribution of $d_{C-C}$ for four systems with indicatively different $\mathrm{F}$ contents $(0.0$, 9.5, 19.1, and 31.0 at.\%, respectively), while Fig. 8 (a, b, c, d) shows the relaxed $\mathrm{CF}_{x}$ structures corresponding to each of these histograms. The histograms shown in Fig. 7 exhibit two main peaks

\footnotetext{
${ }^{2}$ The term $\mathrm{C}-\mathrm{C}$ distance is used to refer to the spacing between any pair of $\mathrm{C}$ atoms and not bond length.
} 


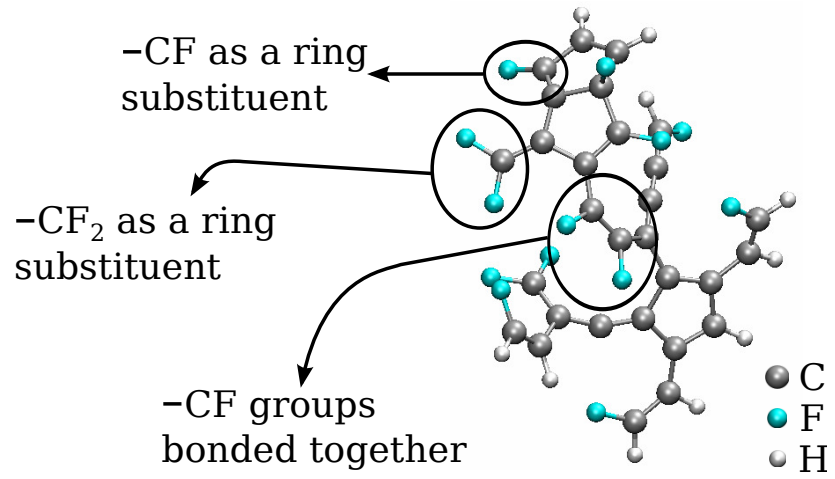

Figure 6: Example of $\mathrm{sp}^{2}$ hybridized $\mathrm{C}$ atoms bonded to $\mathrm{F}$ atoms.

at $\sim 1.4 \AA$ and at $\sim 2.5 \AA$, followed by a large tail representing longer C-C distances. The positions of the first two peaks are consistent with the first two peaks of the radial distribution function determined for different C-based thin films (ta-C, ${ }^{42}$ and $\mathrm{DLC}^{43}$ ) and provide information for the first and second nearest neighbors of the $\mathrm{C}$ atoms in the $\mathrm{CF}_{x}$ films.

As the F content increases, the two first peaks of the $d_{C-C}$ distribution become broader and their position shifts. It is particularly interesting to focus on the evolution of the first peak, since the information from the first nearest neighbor is indicative of short range structure and $\mathrm{C}$ hybridization. To illustrate the evolution with F content of the position of the first peak (in Fig. 7 (a-d), the plot in Fig. 9 shows the average value for the distances to the first nearest neighbors $\left(\bar{d}_{C-C}^{1}\right)$ as a function of $\mathrm{F}$ concentration. For pure carbon systems, the first peak is centered in $\sim 1.42 \AA$ with an average broadening of $0.02 \AA$. With increasing F concentration, two types of behavior can be identified depending on the structural group to which the model system belongs:

(i) for the group of systems characterized by rings arranged in an interlocked pattern, the center of the first peak evolves from being at $\sim 1.42 \AA$ to being at $\sim 1.46 \AA$. This is accompanied by a broadening of the peaks up to $\sim 0.10 \AA$. These values should be compared to the benchmark $\mathrm{C}-\mathrm{C}$ bond lengths in graphene $(1.42 \AA)$, and in diamond $(1.54 \AA)$, and point to an increase in the number of $\mathrm{C}$ atoms with $\mathrm{sp}^{3}$ hybridization.

(ii) On the other hand, the group of systems that are characterized by the prevalence of chains exhibit a shift of the first peak toward shorter $\mathrm{C}-\mathrm{C}$ distances, i.e., from $\sim 1.42 \AA$ to $\sim$ 

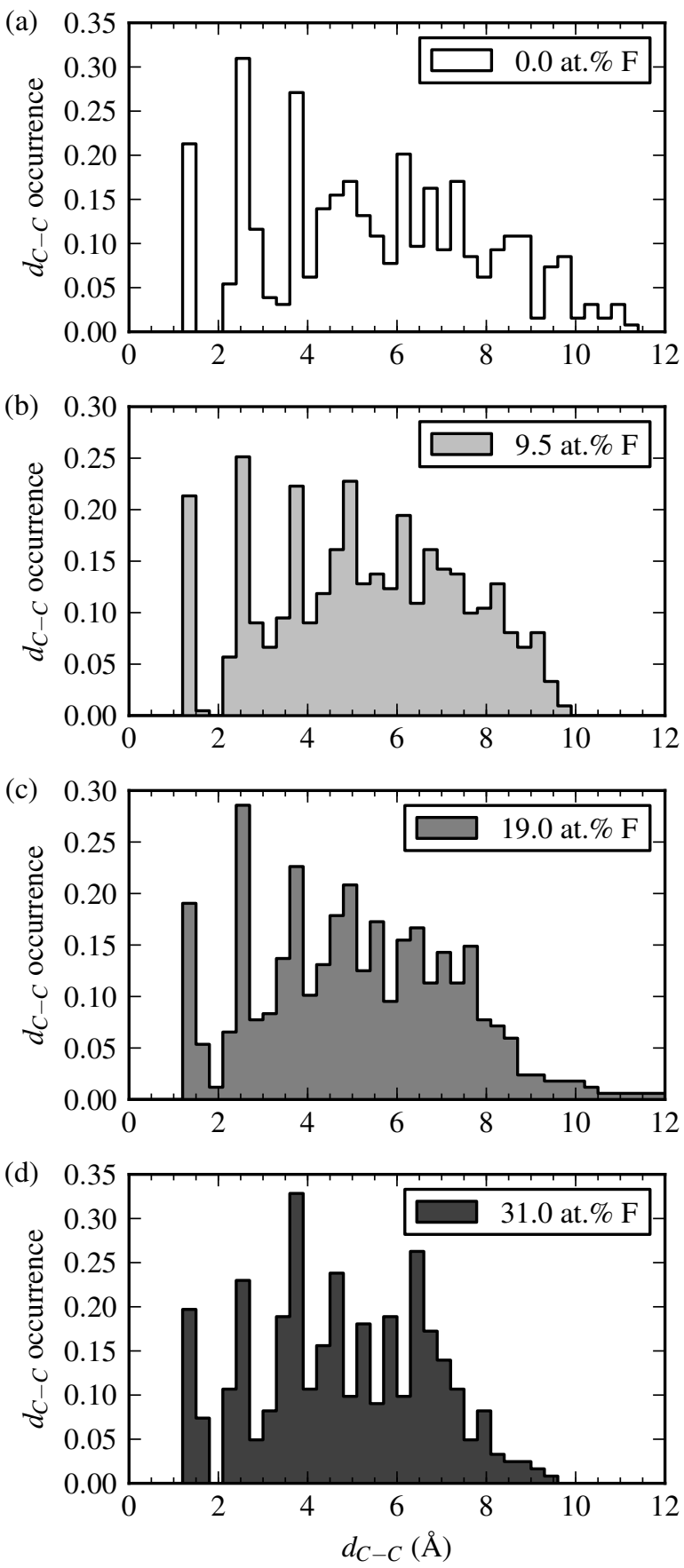

Figure 7: Distribution of $d_{C-C}$ for four systems with different $\mathrm{F}$ content (a) 0.0 at.\%, (b) 9.5 at.\%, (c) 19.0 at.\%, (d) 31.0 at.\%. The number of times a distance between two $\mathrm{C}$ atoms occur in the $\mathrm{CF}_{x}$ system is referred to as " $d_{C-C}$ occurrence" and it is normalized according to the number of $\mathrm{C}$ atoms in the systems. 
(a)

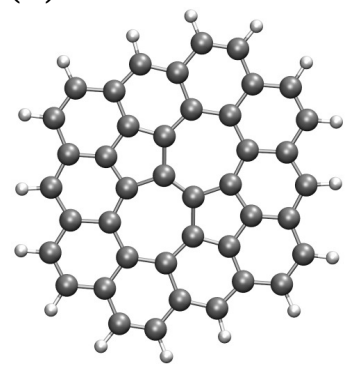

0.0 at. $\%$ (b)

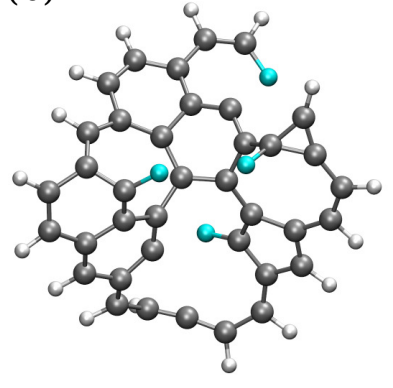

9.5 at. $\%$ (c)

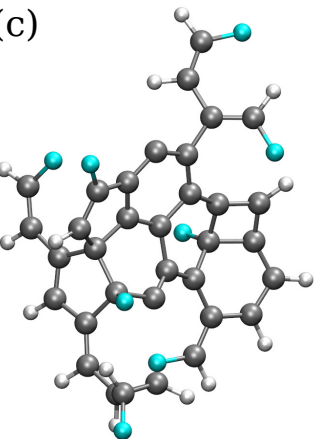

19.0 at. $\%$ (d)

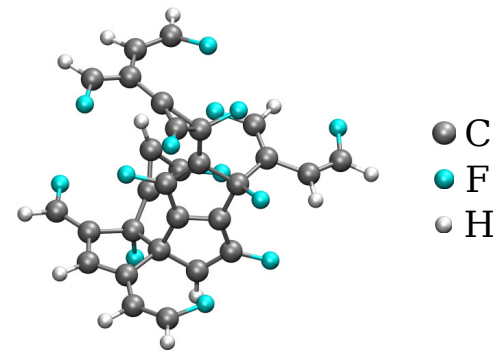

31.0 at. $\%$

Figure 8: $\mathrm{CF}_{x}$ structures corresponding to the histograms presented in Fig. 7. The structures correspond to the following F concentrations: (a) 0.0 at.\%, (b) 9.5 at.\%, (c) 19.0 at.\% and (d) 31.0 at. $\%$, as indicated below each of them, thus, establishing the correspondence to the histograms in Fig. 7.

$1.38 \AA$. Such behavior is related to the formation of polymer-like structures, where the $\mathrm{C}-\mathrm{C}$ bond lengths usually range from $1.35 \AA$ to $1.54 \AA$, depending on the predominating type of bonding.

Since the starting point of all model systems before F-incorporation are graphene-like sheets, the peaks' shifts and also their broadening are an indication for a gradual, but quick transition from highly ordered graphene-like network towards a more disordered structure, a transition which also implies a diversification of the $\mathrm{C}$ atoms hybridization states.

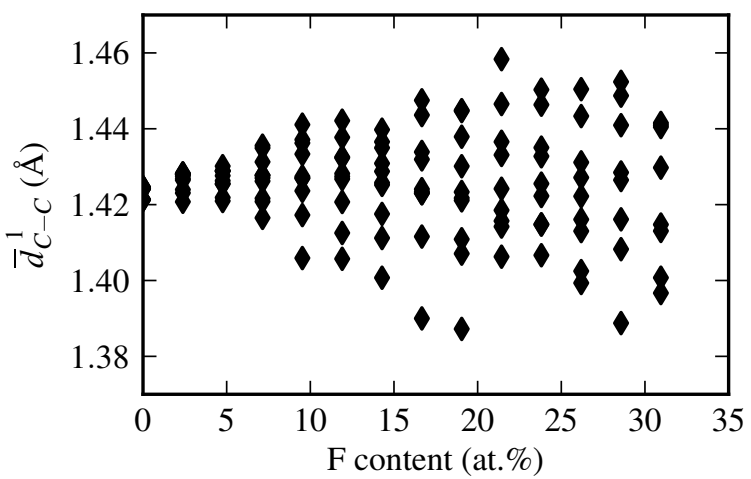

Figure 9: Evolution of the average value for the distances to the first nearest neighbors $\left(\bar{d}_{C-C}^{1}\right)$ as a function of $\mathrm{F}$ concentration.

Additional information can be obtained from the tail in the $d_{C-C}$ distribution plots (Fig. 7 (ad)), especially corresponding to $\mathrm{C}-\mathrm{C}$ distances larger than $2.5 \AA$. In particular, two characteristics 
of the tail can be used to compare ${ }^{3}$ and understand the type of structures that the plots describe: the width of the tail and its discreteness.

The width of the tail indicates the distribution of $d_{C-C}$ in the systems and how spread they are in space: the wider the tail, the more disperse are the $d_{C-C}$. In particular, a wider tail also indicates larger $d_{C-C}$. In the case of systems with the same number of atoms, wider tails point to structures spread in larger space (i.e., systems with lower density). In the current study, the $\mathrm{CF}_{x}$ systems with wider tails have been identified as mostly related to structures characterized by formation of chains. In contrast, narrower tails refer to more compact and dense conformations characterized by the formation of rings possibly intermixed with very short chains.

In particular, one can compare the histograms corresponding to the F concentrations 9.5 at.\% and 19 at.\% in Fig. 7 (b) and (c) respectively. It can be observed that the histogram corresponding to 19 at.\% spreads to larger distances than the one corresponding to 9.5 at.\%. This indicates that the structure with the larger F content is more spread in space than the one with the lower F content, fact that can be observed comparing the structures (b) and (c) in Fig. 8.

The discreteness of the tail can be correlated to the degree of disorder of a system. A tail with higher degree of discreteness refers to a tail with well-defined peaks at certain $d_{C-C}$, without a smooth transition along the $d_{C-C}$ axis. Such type of tail implies that levels of neighbors can be defined, and therefore, a certain degree of structural order characterizes the corresponding $\mathrm{CF}_{x}$ system. On the other hand, a tail with peaks that ascend and descend in a smoother way, without empty spaces between them is an example of low degree of discreteness. which is indicative for a structure with higher degree of disorder reflecting larger variety of bonding and structural diversity.

The significance of the discreteness of the tail can be further understood comparing the histograms corresponding to the F concentrations 0.0 at.\% (Fig. 7 (a)) and 31.0 at.\% (Fig. 7 (d)), respectively. While the first histogram exhibits well defined peaks corresponding to an ordered structure (see Fig. 8 (a), 0.0 at.\%), the histogram corresponding to 31 at.\% of F shows a smoother

\footnotetext{
${ }^{3}$ In order to compare histograms relevant to different systems, they should have the same bin width. Therefore, in the case of systems with different number of atoms, the integral area covered by the histograms bins should also be normalized. This operation was executed for all histograms considered here.
} 
distribution indicating a more disordered $\mathrm{CF}_{x}$ structure (see Fig. 8 (d)).

These properties of the $d_{C-C}$ distribution tail are not directly correlated to the concentration of F. However, they substantially help the description and characterization of the fine structural features of the $\mathrm{CF}_{x}$ compound, since different $\mathrm{F}$ concentrations are reflected in diversity of structures that are associated with a given tail shape.

\section{$\mathrm{CF}_{x}$ structure and $\mathbf{F}$ concentration}

The results discussed in the previous subsections are indicative for the structural features of $\mathrm{CF}_{x}$ films that should be expected at different $\mathrm{F}$ concentrations.

At low F concentrations, up to $\sim 5$ at. $\%$, the graphene-like structure is partially preserved and smooth bending of graphene-like sheets can occur. Therefore, at these F concentrations, elastic films with FL or graphite-like structure are expected. In addition, $\mathrm{C}$ atoms may acquire $\mathrm{sp}^{3}$ hybridization, which would lead to cross-linking of the graphene-like planes leading to an increase in hardness with respect to graphite but this is expected to be still be lower than in the case of DLC films.

The increasing $\mathrm{F}$ content leads to rapid degradation of the 2 dimensional $\mathrm{sp}^{2}$ network by changes in hybridization of the $\mathrm{C}$ atoms, as well as, to an increased density of defects in the network. Above a F content of 10 at. $\%$, there is an increase in the diversity of $\mathrm{CF}_{x}$ structures that can be observed. In particular, many of the relaxed structures at these F concentrations show middle-size rings $(5,6$, and 7 members) arranged in an interlocked manner, thus structurally closely approaching a-C films. These films are expected to have lower elasticity than the previous ones, but an enhanced hardness. For even larger $\mathrm{F}$ contents ( $\geq 15$ at.\%), the $\mathrm{CF}_{x}$ systems tend to form chains, leading to polymer-like structures. The maximum F concentration considered in this work is 31 at. \%, but it is expected that further increasing the F content of the films, polymer-like struc-

tures will predominate. This could result in a Teflon ${ }^{\circledR}$-like material with the additional properties inherent to a FL compound, such as high hardness and high elasticity.

Fig. 10 schematically illustrates the above discussion about the correlation between structure 
and $\mathrm{F}$ content (in the range $0-31$ at.\%) for $\mathrm{CF}_{x}$ films. The color gradient indicates how typical is the considered type of structure for a given F content and should be compared only within the given type of $\mathrm{CF}_{x}$ film. For example, FL structures may develop at really low F concentrations, but as more $\mathrm{F}$ is introduced in the film, FL structure becomes less likely.

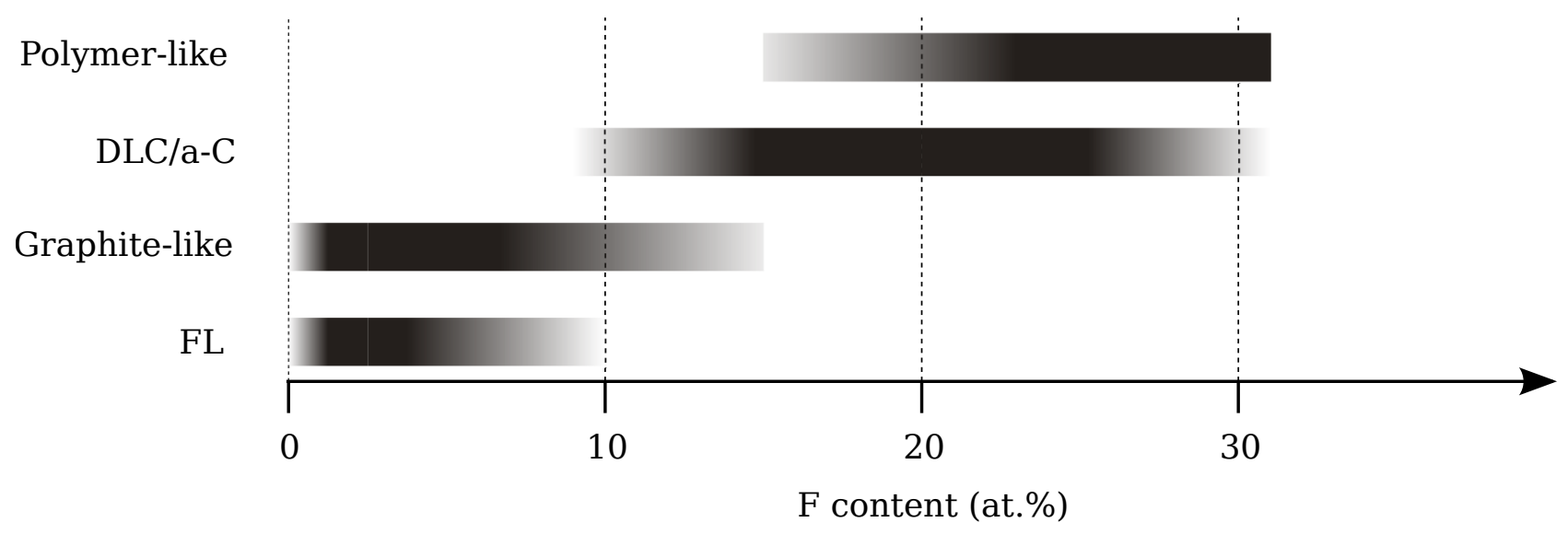

Figure 10: Illustration of the expected type of film depending on the F content. The color gradient is related to how typical the formation of the corresponding structure is for a given $\mathrm{F}$ content.

This span of $\mathrm{CF}_{x}$ films with different structure should be taken together with the consideration of the deposition techniques used for synthesis as well as the corresponding process conditions, such as $\mathrm{C}$ and $\mathrm{F}$ sources, and temperature.

\section{Conclusions}

Fluorinated C-based thin films were addressed by first principles calculations following the SGC approach. The systematic study reveals the interplay between the defects and disruptions resulting from the incorporation of $\mathrm{F}$ atoms in graphene-like networks and how their interaction defines the structural features of these films.

Besides confirming the original predictions from Ref. ${ }^{30}$ regarding $\mathrm{F}$ concentration as a determinant factor for the final structure of $\mathrm{CF}_{x}$ films, by studying the structural evolution and the cohesive energy per atom for 125 different $\mathrm{CF}_{x}$ model systems, the present work answers the question "how" the $\mathrm{F}$ concentration influences the structural characteristics of $\mathrm{CF}_{x}$ compounds. It also 
quantitatively revealed the following trends: (i) for $\mathrm{F}$ concentrations up to $\sim 5$ at. $\%$, the films are expected to exhibit FL or graphite-like features, (ii) the increasing F content opens the possibility of obtaining a large variety of films' structures, including DLC or other types of a-C (at F contents higher than $\sim 10$ at.\%), as well as polymer-like $\mathrm{C}$ films (at $\sim 20$ at.\% of $\mathrm{F}$ and higher concentrations).

The large sample of model systems studied allowed to develop an approach to characterize and predict the structural features of $\mathrm{CF}_{x}$ films based on the statistics of simulation results obtained by SGC. The bonding environment of the $\mathrm{C}$ atoms was determined by studying the relative content of different hybridization states of the $\mathrm{C}$ atoms in $\mathrm{CF}_{x}$ films. It was shown how $\mathrm{F}$ atoms induce changes in $\mathrm{C}$ atoms hybridization and specific groups (such as $-\mathrm{CF}_{2}$ and $-\mathrm{CF}$ ) were identified as responsible for particular structural features of the $\mathrm{CF}_{x}$ (such as polymer-like, fullerene-like or amorphous). The distribution of $\mathrm{C}-\mathrm{C}$ distances provides information for the $\mathrm{CF}_{x}$ films structural disorder, their density and their fine structural features. In particular, it was shown how the width of the $d_{C-C}$ distribution tail is directly related to the spread of the $\mathrm{CF}_{x}$ networks in space, and the discreteness of the tail is indicative of the degree of disorder of the corresponding $\mathrm{CF}_{x}$ structure.

\section{Acknowledgement}

The authors are grateful to Dr. Mattias Jakobsson for facilitating data extraction. C. G. and G.K.G. are grateful for the support by Linköping Linnaeus Initiative on Novel Functionalized Materials (VR) and by FunMat (Functional Nanoscale Materials) - a VINN Excellence Centre (Swedish Agency for Innovation Systems VINNOVA). G.K.G. acknowledges further the support by the Swedish Foundation for Strategic Research (SSF) Synergy Grant \#RMA11-0029 on Functional Carbides and Advanced Surface Engineering (FUNCASE), as well as the Carl Trygger Foundation for Scientific Research.

\section{References}

(1) Lettington, A. H. Carbon 1998, 36, 555-560. 
(2) Ravi, S., Silva, P., Eds. Properties of amorphous carbon; Institution of Engineering and Technology (IET): London, 2002.

(3) Robertson, J. Mater Sci Eng R 2002, 37, 129-281.

(4) Hultman, L.; Neidhardt, J.; Hellgren, N.; Sjöström, H.; Sundgren, J.-E. MRS Bull 2003, 28, 194-202.

(5) Grill, A. Diam Relat Mater 1999, 8, 428-434.

(6) Kunze, T.; Gemming, S.; Posselt, M.; Seifert, G. Zeitschrift für Physikalische Chemie 2011, $225,379-387$.

(7) Wang, P.; Wang, X.; Zhang, B.; Liu, W. Thin Solid Films 2010, 518, 5938-5943.

(8) Kundoo, S.; Saha, P.; Chattopadhyay, K. Mater Lett 2004, 58, 3920-3924.

(9) McCann, R.; Roy, S. S.; Papakonstantinou, P.; McLaughlin, J. a.; Ray, S. C. J Appl Phys 2005, 97, 073522.

(10) Sjöström, H.; Stafström, S.; Boman, M.; Sundgren, J.-E. Phys Rev Lett 1995, 75, 1336-1339.

(11) Furlan, A.; Gueorguiev, G. K.; Högberg, H.; Stafström, S.; Hultman, L. Thin Solid Films 2006, 515, 1028-1032.

(12) Gueorguiev, G. K.; Furlan, A.; Högberg, H.; Stafström, S.; Hultman, L. Chem Phys Lett 2006, 426, 374-379.

(13) Furlan, A.; Gueorguiev, G. K.; Czigány, Z.; Högberg, H.; Braun, S.; Stafström, S.; Hultman, L. phys status solidi (RRL) 2008, 2, 191-193.

(14) Moolsradoo, N.; Watanabe, S. Advances in Materials Science and Engineering 2010, 2010, $1-7$. 
(15) Goyenola, C.; Gueorguiev, G. K.; Stafström, S.; Hultman, L. Chem Phys Lett 2011, 506, 86-91.

(16) Goyenola, C.; Stafström, S.; Hultman, L.; Gueorguiev, G. K. J Phys Chem C 2012, 116, 21124-21131.

(17) Endo, K.; Shinoda, K.; Tatsumi, T. J Appl Phys 1999, 86, 2739-2745.

(18) Touhara, H.; Okino, F. Carbon 2000, 38, 241-267.

(19) Agraharam, S.; Hess, D. W.; Kohl, P. A.; Bidstrup Allen, S. A. J Vac Sci Tech B 2001, 19, 439-446.

(20) Jung, H.-s.; Park, H.-h. Thin Solid Films 2002, 420-421, 248-252.

(21) Bendavid, A.; Martin, P.; Randeniya, L.; Amin, M.; Rohanizadeh, R. Diam Relat Mater 2010, 19, 1466-1471.

(22) Tang, G.; Ma, X.; Sun, M.; Li, X. Carbon 2005, 43, 345-350.

(23) Ishihara, M.; Suzuki, M.; Watanabe, T.; Nakamura, T.; Tanaka, A.; Koga, Y. Diam Relat Mater 2005, 14, 989-993.

(24) Yun, Y.; Broitman, E.; Gellman, A. J. Langmuir 2010, 26, 908-914.

(25) Schmidt, S.; Greczynski, G.; Goyenola, C.; Gueorguiev, G.; Czigány, Z.; Jensen, J.; Ivanov, I.; Hultman, L. Surf Coat Technol 2011, 206, 646-653.

(26) Schmidt, S. Carbon nitride and carbon fluoride thin films prepared by HiPIMS. Ph.D. thesis, Linköping University, 2013.

(27) Schmidt, S.; Goyenola, C.; Gueorguiev, G.; Jensen, J.; Greczynski, G.; Ivanov, I.; Czigány, Z.; Hultman, L. Thin Solid Films 2013, 542, 21-30. 
(28) Gueorguiev, G. K.; Neidhardt, J.; Stafström, S.; Hultman, L. Chem Phys Lett 2005, 410, $228-234$.

(29) Gueorguiev, G. K.; Broitman, E.; Furlan, A.; Stafström, S.; Hultman, L. Chem Phys Lett 2009, 482, 110-113.

(30) Gueorguiev, G.; Goyenola, C.; Schmidt, S.; Hultman, L. Chem Phys Lett 2011, 516, 62-67.

(31) Frisch, M. J. et al. Gaussian 09. 2009.

(32) Perdew, J. P.; Jackson, K. A.; Pederson, M. R.; Singh, D. J.; Fiolhais, C. Phys Rev B 1992, 46, 6671-6687.

(33) Becke, A. D. J Chem Phys 1993, 98, 5648-5652.

(34) Stafström, S.; Hultman, L.; Hellgren, N. Chem Phys Lett 2001, 340, 227-231.

(35) Xie, R.-H.; Bryant, G. W.; Jensen, L.; Zhao, J.; Smith, V. H. J Chem Phys 2003, $118,8621$.

(36) Osuna, S.; Torrent-Sucarrat, M.; Sola, M.; Geerlings, P.; Ewels, C.; Lier, G. J Phys Chem C 2010, 114, 3340-3345.

(37) Ignatieva, L.; Bouznik, V. J Fluorine Chem 2011, 132, 724-731.

(38) Grimme, S. J Comp Chem 2006, 27, 1787-99.

(39) Denis, P. A. Chem Phys Lett 2010, 492, 251-257.

(40) Denis, P. A.; Faccio, R.; Mombru, A. W. Chemphyschem 2009, 10, 715-722.

(41) Neidhardt, J. Fullerene-like carbon nitride thin solid films. Ph.D. thesis, Linköping University, 2004.

(42) Gilkes, K.; Gaskell, P.; Robertson, J. Phys Rev B 1995, 51, 12303-12312.

(43) Zhong, Z.; Wang, X.; Feng, X. J Mater Res 2011, 22, 2770-2775. 


\section{Graphical TOC entry}

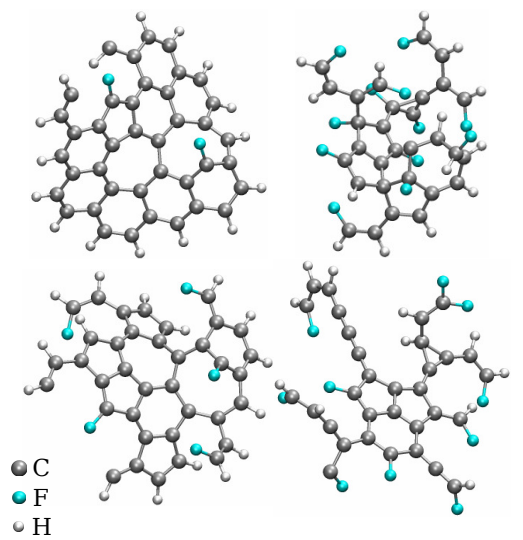

